FUNCTION SPACES XII

BANACH CENTER PUBLICATIONS, VOLUME 119

INSTITUTE OF MATHEMATICS

POLISH ACADEMY OF SCIENCES

WARSZAWA 2019

\title{
ON THE BISHOP-PHELPS-BOLLOBÁS PROPERTY
}

\author{
MARÍA D. ACOSTA \\ Universidad de Granada, Facultad de Ciencias \\ Departamento de Análisis Matemático \\ 18071 Granada, Spain \\ E-mail:dacosta@ugr.es
}

Dedicated to the memory of Bernardo Cascales and Henryk Hudzik

\begin{abstract}
The Bishop-Phelps Theorem states the denseness of the set of norm attaining functionals in the topological dual of any Banach space. Bollobás obtained a quantitative version of this result showing that a pair $\left(x, x^{*}\right)$ given by an element $x$ in the unit sphere of a Banach space and a functional $x^{*}$ in the unit sphere of the dual such that $x^{*}(x)$ is close to 1 can be approximated in norm by another pair $\left(y, y^{*}\right)$ satisfying the same conditions and also that $y^{*}$ attains its norm at $y$. In 2008 the problem of obtaining versions of Bollobás result for operators was considered. In this survey we include most of the results on this topic to the present day.
\end{abstract}

1. Introduction. Throughout this paper $X^{*}$ is the topological dual of a Banach space $X$. By $B_{X}$ and $S_{X}$ we denote the closed unit ball and the unit sphere of $X$, respectively. The symbol $L(X, Y)$ denotes the space of (linear and bounded) operators between two Banach spaces $X$ and $Y$, endowed with the usual operator norm. Bishop and Phelps proved in 1961 that for any Banach space the set of continuous and linear functionals attaining their norms is norm dense in the topological dual [22]. Bollobás [23] obtained in 1970 the following quantitative version of the previous result.

Bishop-Phelps-Bollobás Theorem ([24, Theorem 16.1]). Let $X$ be a Banach space and $0<\varepsilon<1$. Given $x \in B_{X}$ and $x^{*} \in S_{X^{*}}$ with $\left|1-x^{*}(x)\right|<\frac{\varepsilon^{2}}{4}$, there are elements $y \in S_{X}$ and $y^{*} \in S_{X^{*}}$ such that $y^{*}(y)=1,\|y-x\|<\varepsilon$ and $\left\|y^{*}-x^{*}\right\|<\varepsilon$.

2010 Mathematics Subject Classification: Primary 46B04; Secondary 46B28.

Key words and phrases: Banach space, operator, Bishop-Phelps-Bollobás theorem, BishopPhelps-Bollobás property.

The paper is in final form and no version of it will be published elsewhere. 
The paper [30] contains an improvement of the previous result. In Corollary 2.4 the authors show that it suffices to assume that $\left|1-x^{*}(x)\right|<\frac{\varepsilon^{2}}{2}$ to obtain the same assertion.

Bishop and Phelps posed in [22] the problem of extending their result to operators. In 1963 Lindenstrauss exhibited a counterexample showing that in general the set of norm attaining operators is not dense in the space of bounded and linear operators [57]. He also provided assumptions on a Banach space in order that the set of norm attaining operators is dense in the space of linear and bounded operators. For instance, if $X$ is a reflexive Banach space then the set of norm attaining operators from $X$ to $Y$ is dense in $L(X, Y)$, for any Banach space $Y$ [57, Theorem 1]. In 1977 Bourgain proved the parallel result when $X$ has the Radon-Nikodým property [25]. Afterwards several authors proved interesting results and there are also many open problems in the subject. The survey [2] contains some interesting results about the topic and the state of the art until 2006.

This survey deals with versions of the Bishop-Phelps-Bollobás theorem for operators and some other mappings. Let us mention that this result has been useful to obtain properties for numerical ranges of operators (see for instance [24, §17]). In 2008 Acosta, Aron, García and Maestre introduced the following notion.

Definition 1.1 ([4, Definition 1.1]). Let $X$ and $Y$ be both either real or complex Banach spaces. The pair $(X, Y)$ is said to have the Bishop-Phelps-Bollobás property for operators (BPBp) if for every $0<\varepsilon<1$ there exists $0<\eta(\varepsilon)<\varepsilon$ with the following property:

If $T \in S_{L(X, Y)}$ and $x_{0} \in S_{X}$ satisfy $\left\|T\left(x_{0}\right)\right\|>1-\eta(\varepsilon)$, then there exist $S \in S_{L(X, Y)}$ and $u_{0} \in S_{X}$ satisfying the conditions

$$
\left\|S\left(u_{0}\right)\right\|=1, \quad\left\|u_{0}-x_{0}\right\|<\varepsilon \quad \text { and } \quad\|S-T\|<\varepsilon .
$$

The pair $(X, Y)$ has the Bishop-Phelps property if the set of norm attaining operators from $X$ to $Y$ is dense in $L(X, Y)$.

As we already mentioned, there are Banach spaces $X$ and $Y$ such that the pair $(X, Y)$ does not have the Bishop-Phelps property. In such cases the pair $(X, Y)$ does not have the BPBp. In general these two properties are very different.

After the first paper that we mentioned many results have been obtained. Let us say only that there are some geometric properties both on the domain and on the range implying the BPBp. For instance, a certain geometrical property on $Y$, called property $\beta$ of Lindenstrauss (see Definition 2.2), implies that the pair $(X, Y)$ has the Bishop-PhelpsBollobás property for operators for any Banach space $X$. If a Banach space $X$ is uniformly convex, then the pair $(X, Y)$ has the Bishop-Phelps-Bollobás property for operators for any Banach space $Y$. We will include such results in Section 2. We devote the next two sections to results on this topic for classical Banach spaces. Section 3 contains results where the domain space is an $L_{1}$ space and Section 4 deals with the case when the domain is $C(K)$ for some compact Hausdorff topological space $K$. Section 5 is devoted to results for $n$-linear mappings and in Section 6 we examine the Bishop-Phelps-Bollobás property for numerical radius of operators. Finally we include in Section 7 some open problems on the topic. 


\section{Geometric or isomorphic properties implying the Bishop-Phelps-Bollobás} property for operators. A compactness argument yields the following positive result.

Theorem 2.1 (4, Proposition 2.4]). The pair $(X, Y)$ has the BPBp when $X$ and $Y$ are finite-dimensional normed spaces.

We recall a property used by Lindenstrauss [57, Proposition 3] on the range to get denseness of the set of norm attaining operators.

Definition 2.2. A Banach space $Y$ is said to have property $\beta$ (of Lindenstrauss) if there are two sets $\left\{y_{\alpha}: \alpha \in \Lambda\right\} \subset S_{Y},\left\{y_{\alpha}^{*}: \alpha \in \Lambda\right\} \subset S_{Y^{*}}$ and $0 \leq \rho<1$ such that the following conditions hold:

1) $y_{\alpha}^{*}\left(y_{\alpha}\right)=1$.

2) $\left|y_{\alpha}^{*}\left(y_{\beta}\right)\right| \leq \rho<1$ if $\alpha \neq \beta$.

3) $\|y\|=\sup _{\alpha}\left\{\left|y_{\alpha}^{*}(y)\right|\right\}$ for all $y \in Y$.

In the first paper about the topic, the following result was shown.

Theorem 2.3 ([4, Theorem 2.2]). Let $X$ and $Y$ be Banach spaces such that $Y$ has property $\beta$. Then the pair $(X, Y)$ has the Bishop-Phelps-Bollobás property for operators. Indeed, if $T \in S_{L(X, Y)}, \varepsilon>0$ and $x_{0} \in S_{X}$ satisfy $\left\|T\left(x_{0}\right)\right\|>1-\frac{\varepsilon^{2}}{4}$, then for each real number $\eta$ such that $\eta>\frac{\rho}{1-\rho}\left(\varepsilon+\frac{\varepsilon^{2}}{4}\right)$, there are $S \in L(X, Y), z_{0} \in S_{X}$ such that

$$
\left\|S z_{0}\right\|=\|S\|, \quad\left\|z_{0}-x_{0}\right\|<\varepsilon, \quad\|S-T\|<\eta+\varepsilon+\frac{\varepsilon^{2}}{4} .
$$

Roughly speaking, the idea of the proof of this result is to apply condition 3) in Definition 2.2 to the element $T\left(x_{0}\right)$ to get a convenient functional $y_{\alpha}^{*}$ and then apply the Bishop-Phelps-Bollobás theorem to the functional $T^{*}\left(y_{\alpha}^{*}\right)$ to define a new operator in $L(X, Y)$ whose difference with $T$ is a rank-one operator.

Let us notice that a finite-dimensional space has property $\beta$ if and only if its unit ball is a polyhedron. The spaces $c_{0}$ and $\ell_{\infty}$, endowed with their usual norms, have property $\beta$ of Lindenstrauss. In general, if $K$ is a compact Hausdorff topological space, $C(K)$ has this property when $K$ has a dense set of isolated points. Under isomorphisms, property $\beta$ is not restrictive at all since every Banach space admits an equivalent norm with this property [58].

In order to state a sufficient condition on the domain implying the BPBp, we recall that a Banach space $X$ is uniformly convex if for every $\varepsilon>0$ there is $0<\delta<1$ such that for all $u, v \in B_{X}$ such that $\frac{\|u+v\|}{2}>1-\delta$, we have $\|u-v\|<\varepsilon$.

In such a case, the modulus of convexity of $X$ is given by

$$
\delta_{X}(\varepsilon):=\inf \left\{1-\frac{\|u+v\|}{2}: u, v \in B_{X},\|u-v\| \geq \varepsilon\right\} \quad(\varepsilon \in] 0,2[) .
$$

The following result has been obtained independently by several authors. It was proved by Kim and Lee in [50, Theorem 3.1] and also by Acosta, Becerra-Guerrero, García and Maestre in [10, Theorem 2.2]. Both papers provide different estimates of the function $\eta$ appearing in Definition 1.1 . 
TheOrem 2.4. Let $X$ and $Y$ be Banach spaces and assume that $X$ is uniformly convex. Given $\varepsilon>0$, if $0<\eta<\delta_{X}(\varepsilon) \frac{\varepsilon}{8+2 \varepsilon}$, then for every $T \in S_{L(X, Y)}$ and every $x_{0} \in S_{X}$ such that $\left\|T\left(x_{0}\right)\right\|>1-\eta$, there are a point $z_{0} \in S_{X}$ and $S \in S_{L(X, Y)}$ satisfying the following conditions:

$$
\left\|S\left(z_{0}\right)\right\|=1, \quad\left\|z_{0}-x_{0}\right\| \leq \varepsilon \quad \text { and } \quad\|S-T\|<\varepsilon .
$$

The proofs provided in the mentioned two papers are very different. In [50] the authors define a sequence of operators in $L(X, Y)$ such that the difference between two consecutive terms is a rank-one operator in order to obtain the desired operator $S$. In the case of the paper [10] the authors use a perturbation result due to Stegall and valid for spaces with the Radon-Nikodým property. We also remark that in the first paper mentioned the authors obtain a characterization of uniformly convex spaces in terms of an assertion stronger than the Bishop-Phelps-Bollobás theorem [50, Theorem 2.1].

For the case of a Hilbert space, García, Lee and Maestre proved the following result.

TheOREM 2.5 ([41, Theorem 2.1]). The class of self-adjoint operators on any complex Hilbert space satisfy the Bishop-Phelps-Bollobás property.

To finish this section, we mention that there are several papers containing different estimates of Bishop-Phelps-Bollobás moduli for the scalar case [30, 29, 28, 47, 43] and also for operators 48 .

3. Results when the domain is an $L_{1}$ space. If $Y$ is a Banach space, an element $T \in L\left(\ell_{1}, Y\right)$ can be identified with the sequence $\left\{T\left(e_{n}\right)\right\}$ of elements in $Y$ satisfying $\sup \left\{\left\|T\left(e_{n}\right)\right\|: n \in \mathbb{N}\right\}=\|T\|$. It is elementary to check that the operator $T$ in $L\left(\ell_{1}, Y\right)$ attains its norm if and only if the norm of one of the terms of the associated sequence is the largest norm of all the terms. With this argument it is very easy to show the denseness of the set of norm attaining operators from $\ell_{1}$ to $Y$ in the space $L\left(\ell_{1}, Y\right)$. However, in general the pair $\left(\ell_{1}, Y\right)$ does not have the Bishop-Phelps-Bollobás property for operators. In the paper [4 the authors obtained a characterization of the Banach spaces $Y$ such that $\left(\ell_{1}, Y\right)$ satisfies the BPBp. The property appearing in this characterization is quite technical and was introduced in [4, Definition 3.1]. Afterwards another geometric property related to the same topic was used and called the approximate hyperplane property (see [34, Definition 2.1]). We recall both properties.

Definition 3.1. A Banach space $X$ has the approximate hyperplane property (AHp) if there exists a function $\delta:] 0,1[\longrightarrow] 0,1\left[\right.$ and a 1-norming subset $C$ of $S_{X^{*}}$ satisfying the following property:

Given $\varepsilon>0$, there is a function $\Upsilon_{X, \varepsilon}: C \longrightarrow S_{X^{*}}$ with the condition

$$
x^{*} \in C, x \in S_{X}, \operatorname{Re} x^{*}(x)>1-\delta(\varepsilon) \Rightarrow \operatorname{dist}\left(x, F\left(\Upsilon_{X, \varepsilon}\left(x^{*}\right)\right)\right)<\varepsilon,
$$

where $F\left(y^{*}\right)=\left\{y \in S_{X}: \operatorname{Re} y^{*}(y)=1\right\}$ for any $y^{*} \in S_{X^{*}}$.

A family of Banach spaces $\left\{X_{i}: i \in I\right\}$ has $A H p$ uniformly if every space $X_{i}$ has property AHp with the same function $\delta$.

In what follows by a convex series we mean a series $\sum \alpha_{n}$, where $0 \leq \alpha_{n} \leq 1$ for each $n \in \mathbb{N}$ and $\sum_{n=1}^{\infty} \alpha_{n}=1$. 
Definition 3.2. A Banach space $X$ has the approximate hyperplane series property (AHSp) if and only if for every $0<\varepsilon<1$ there exists $0<\eta<\varepsilon$ such that for every sequence $\left\{x_{n}\right\}$ in $S_{X}$ and every convex series $\sum \alpha_{n}$ with

$$
\left\|\sum_{n=1}^{\infty} \alpha_{n} x_{n}\right\|>1-\eta,
$$

there exist a subset $A \subset \mathbb{N}$ and a subset $\left\{z_{k}: k \in A\right\} \subset S_{X}$ satisfying

1) $\sum_{k \in A} \alpha_{k}>1-\varepsilon$

2) $\left\|z_{k}-x_{k}\right\|<\varepsilon$ for all $k \in A$,

3) there is $x^{*} \in S_{X^{*}}$ such that $x^{*}\left(z_{k}\right)=1$ for every $k \in A$.

Some characterizations of the AHSp can be found in [5, Proposition 1.2]. It is known that AHp implies AHSp [34, Proposition 2.2] and most of the spaces with the second property indeed have the AHp. In fact, it is not known if the two properties are equivalent.

The following classes of spaces have the AHp:

1) uniformly convex spaces,

2) finite-dimensional spaces,

3) separable lush spaces (see [34, p. 246] for the definition of lush space),

4) almost CL-spaces,

5) spaces with the property $\beta$ of Lindenstrauss.

As a consequence, $C(K)$ and $L_{1}(\mu)$ have the AHp for any compact and Hausdorff topological space $K$ and any positive measure $\mu$ since they are CL-spaces. All the results providing spaces with the AHp that we listed can be found in [34, Section 2] and some of them appear implicitly in [4. Moreover, if $X$ is a Banach space satisfying some of the properties included above, the space $L_{1}(\mu, X)$ also has the AHp [34, Corollary 2.12]. It is also known that $\mathrm{AHp}$ is not stable under infinite $c_{0}, \ell_{1}$ and $\ell_{\infty}$ sums. The spaces $L(X, \mathcal{C}(K))$ and $\mathcal{K}(X, \mathcal{C}(K))$ have AHSp for every uniformly smooth Banach space $X$ and every compact Hausdorff topological space $K$ [8, Corollary 4.4].

Let us mention that Choi and Kim proved that lush spaces have AHSp [32, Theorem 7]. This result extends one for CL-spaces shown by Cheng, Dai and Dong [27, Theorem 2.1]. Also the space $L_{1}(\mu, X)$ has the approximate hyperplane series property for any $\sigma$-finite measure $\mu$ whenever $X$ is uniformly convex [32, Theorem 14].

The main result that was the motivation to introduce property AHSp is the following characterization.

TheOREm 3.3 ([4, Theorem 4.1]). For a Banach space $Y$, the pair $\left(\ell_{1}, Y\right)$ has the BishopPhelps-Bollobás property for operators if and only if $Y$ satisfies AHSp.

The argument in the proof of the previous result uses the fact that an element $T \in S_{L\left(\ell_{1}, Y\right)}$ can be identified with the sequence $\left\{T\left(e_{n}\right)\right\}$ of elements in $Y$ satisfying $\sup \left\{\left\|T\left(e_{n}\right)\right\|: n \in \mathbb{N}\right\}=1$. In this way an element $x_{0} \in S_{\ell_{1}}$ such that $\left\|T\left(x_{0}\right)\right\|$ is close to 1 provides, up to a linear surjective isometry on $\ell_{1}$, a convex series $\sum x_{0}(n) T\left(e_{n}\right)$ in $Y$, whose norm is also close to 1 . This is the idea connecting the fact that $T$ almost attains its norm at $x_{0}$ with the condition that appears in the definition of AHSp. If one assumes that the space has AHSp, the operator $S$ defined by $S\left(e_{n}\right)=T\left(e_{n}\right)$ for $n \in \mathbb{N} \backslash A$ and 
$S\left(e_{n}\right)=z_{n}$ for $n \in A$, where $\left\{z_{n}: n \in A\right\}$ is the subset appearing in Definition 3.2 for the sequence $\left\{x_{n}\right\}=\left\{T\left(e_{n}\right)\right\}$, is the operator that we want in order to show that the pair $\left(\ell_{1}, Y\right)$ has the Bishop-Phelps-Bollobás property for operators.

In general, there are a few stability results for pairs $(X, Y)$ such that the set of norm attaining operators from $X$ to $Y$ is dense in the space $L(X, Y)$. Indeed it is a longstanding open problem if the pair $\left(X, \mathbb{R}^{2}\right)$ satisfies the previous property, where $\mathbb{R}^{2}$ has the euclidean norm. Let us also notice that on the range one cannot expect too many stability results since there are counterexamples for infinite $\ell_{p}$-sums for $1 \leq p<+\infty$ (see [45. Theorem, p. 149] and [1, Theorem 2.3]).

However, the following stability results of the Bishop-Phelps-Bollobás property have been obtained.

Proposition 3.4. The following assertions are satisfied:

1) The pair $\left(X,\left(\bigoplus \sum_{n=1}^{\infty} Y_{n}\right)_{c_{0}}\right)$ and $\left(X,\left(\bigoplus \sum_{n=1}^{\infty} Y_{n}\right)_{\ell_{\infty}}\right)$ satisfy the Bishop-PhelpsBollobás property for operators whenever all pairs $\left(X, Y_{n}\right)$ have the Bishop-PhelpsBollobás property for operators "uniformly".

2) If $X_{i}$ is a Banach space with the AHSp for each $1 \leq i \leq N$, then the space $\prod_{i=1}^{N} X_{i}$, endowed with some normalized absolute norm, also has the AHSp. We recall that an absolute norm on a finite cartesian product is a norm that depends only on the norms of all the components. This norm is normalized if the natural embedding of each space $X_{i}$ in the cartesian product is a linear isometry.

3) If $E$ is a Banach sequence lattice with the AHSp that is uniformly monotone and $\left\{X_{k}: k \in \mathbb{N}\right\}$ is a family of Banach spaces with the AHp uniformly, then $\left(\oplus X_{n}\right)_{E}$ has the AHSp.

The first statement of the result was proved in [20, Proposition 2.4], where some stability results on the domain can also be found. The result in 2) appears in [16] and has been obtained previously in [5. Corollary 2.8] for $\ell_{p}$-sums of two Banach spaces for $1 \leq p<\infty$ and in [15, Theorem 2.6] for any absolute norm on the product of two spaces. Finally the result 3) appeared in [15, Theorem 2.10]. Section 2 in [15] contains also all the notions used in the last two assertions.

We also remark that the results in 1) and 2) are indeed characterizations. These facts have been essentially proved in [20, Proposition 2.4], 42, Theorem 2.3] and [15, Theorem $2.6]$.

In [54] Kim, Lee and Martín characterized the pairs of Banach spaces $(X, Y)$ such that $\left(\ell_{1}(X), Y\right)$ satisfies the Bishop-Phelps-Bollobás property for operators [54, Theorem 6]. As a consequence they proved the next result.

Proposition 3.5. In the following cases the pair of spaces $(X, Y)$ is such that $\left(\ell_{1}(X), Y\right)$ has the BPBp:

1) $X$ and $Y$ are finite-dimensional spaces,

2) $Y$ is a Hilbert space and the pair $(X, H)$ has the Bishop-Phelps-Bollobás property for operators,

3) $X=C(K)$ for some Hausdorff and compact topological space $K$ and $Y$ is a Hilbert space. 
The following extension of 2) has been obtained. If $\left\{X_{i}: i \in I\right\}$ is a family of Banach spaces, $H$ is a Hilbert space such that the pair $\left(X_{i}, H\right)$ has the BPBp for operators for every $i \in I$ with the same function $\eta$, then the pair $\left(\left(\oplus \sum_{i \in I} X_{i}\right)_{\ell_{1}}, H\right)$ has the BPBp [15. Proposition 2.3].

As we already mentioned, there is a characterization of the spaces $Y$ such that the pair $\left(\ell_{1}, Y\right)$ has the Bishop-Phelps-Bollobás property for operators that we stated explicitly. However this is not the case when the domain is any $L_{1}$ space. So we will include the known results in this case.

Let us say that when the domain $X$ is a finite-dimensional $L_{1}$ space, as a consequence of the argument used in [4, Theorem 4.1], there is a characterization of the spaces $Y$ such that the pair $(X, Y)$ has the BPBp. If $X$ has dimension $N$ and it is an $L_{1}$ space, then the pair $(X, Y)$ has the BPBp if $Y$ satisfies Definition 3.2 for convex combinations of $N$ elements instead of convex series.

So we can consider only infinite-dimensional $L_{1}$ spaces on the domain. The first result proved by Choi and Kim on this topic essentially solves the problem in case that the range is a space with the Radon-Nikodým property.

ThEOREM 3.6 ([31, Theorem 2.2]). Assume that $Y$ is a Banach space with the RadonNikodým property and $\mu$ a $\sigma$-finite measure such that $L_{1}(\mu)$ is infinite-dimensional. Then the pair $\left(L_{1}(\mu), Y\right)$ has the Bishop-Phelps-Bollobás property for operators if and only if $Y$ has the approximate hyperplane series property.

The proof of the previous result uses in an essential way that under the assumptions an operator from $L_{1}(\mu)$ to $Y$ can be represented by a function in $L_{\infty}(\mu, Y)$.

Let us notice that in general for any Banach space $Y$, in case that $\mu$ is any positive measure such that $L_{1}(\mu)$ is infinite-dimensional, if the pair $\left(L_{1}(\mu), Y\right)$ has the BishopPhelps-Bollobás property for operators, then $Y$ has the approximate hyperplane series property (see [31, Theorem 2.1] and [8, Proposition 2.2]). Because of results that we will mention later it is known that the Radon-Nikodým property is not needed in order that the pair $\left(L_{1}(\mu), Y\right)$ has the BPBp.

For some classical spaces in the range there are also positive results.

THEOREM 3.7. Let $\mu$ and $\nu$ be positive measures. Then the following assertions are satisfied:

1) The pair $\left(L_{1}(\mu), L_{1}(\nu)\right)$ has the Bishop-Phelps-Bollobás property for operators.

2) If $\nu$ is a localizable measure, then the pair $\left(L_{1}(\mu), L_{\infty}(\nu)\right)$ has the BPBp.

Both results have been proved by Choi, Kim, Lee and Martín [33, Theorems 3.1 and 4.1]. The second assertion was shown before by Aron, Choi, García and Maestre for the pair $\left(L_{1}(\mu), L_{\infty}[0,1]\right)$ when $\mu$ is a $\sigma$-finite measure [19]. Section 5 in [33] also contains positive results for certain classes of operators in $L\left(L_{1}(\mu), C(K)\right)$ in the real case, for any positive finite measure $\mu$ and any compact Hausdorff topological space $K$.

Inspired by the results in [31, Acosta, Becerra-Guerrero, Kim, García and Maestre also obtained some positive results about the Bishop-Phelps-Bollobás property for some classes of operators when the domain is an $L_{1}$ space. For this, they introduced the following notion. 
Definition 3.8 ([8, Definition 1.3]). Let $X$ and $Y$ be both real or complex Banach spaces and $M$ a subspace of $L(X, Y)$. We say that $M$ satisfies the Bishop-Phelps-Bollobás property if given $\varepsilon>0$, there is $\eta(\varepsilon)>0$ such that for any $T \in S_{M}$, if $x_{0} \in S_{X}$ satisfies $\left\|T x_{0}\right\|>1-\eta(\varepsilon)$, then there exist a point $u_{0} \in S_{X}$ and an operator $S \in S_{M}$ satisfying the following conditions:

$$
\left\|S u_{0}\right\|=1, \quad\left\|u_{0}-x_{0}\right\|<\varepsilon \quad \text { and } \quad\|S-T\|<\varepsilon .
$$

Proposition 3.9 ([8, Proposition 2.2]). Let $(\Omega, \Sigma, \mu)$ be a measure space such that $L_{1}(\mu)$ is infinite-dimensional, $Y$ a Banach space, and $M$ a subspace of $L\left(L_{1}(\mu), Y\right)$ containing all finite-rank operators. If $M$ has the Bishop-Phelps-Bollobás property, then $Y$ has the approximate hyperplane series property.

The converse result also holds true for certain classes of subspaces $M$ of $L\left(L_{1}(\mu), Y\right)$. TheOrem 3.10 ([8, Theorem 2.3]). Let $(\Omega, \Sigma, \mu)$ be a finite measure space, $Y$ a Banach space with $A H S p$ and $M$ a subspace of $\mathcal{L}\left(L_{1}(\mu), Y\right)$ that contains all finite-rank operators and is contained in the subspace of all representable operators. Also, assume that the operator $S_{A}(f)=S\left(f \chi_{A}\right)$ belongs to $M$ whenever $S \in M$ and $A$ is any measurable subset of $\Omega$. Then $M$ has the Bishop-Phelps-Bollobás property for operators.

As a consequence, under the previous assumptions on the measure space, if $Y$ is a Banach space, then the space of finite-rank operators has the Bishop-Phelps-Bollobás property precisely when $Y$ has the approximate hyperplane series property. Instead of the subspace of finite-rank operators, the ideal of compact operators or the subspace of weakly compact operators can be used (see [8, Corollary 2.4]).

\section{Results about the Bishop-Phelps-Bollobás property for operators when} the domain is $C(K)$. The purpose of this section is to state the results known about the topic when the domain is $C(K)$. In this case the problem seems to be quite complicated even for finite-dimensional spaces. The first result of this type was obtained in [4. Theorem 5.2] for operators from $\ell_{\infty}^{n}$ to a uniformly convex Banach space. Here $\ell_{\infty}^{n}$ denotes the space $\mathbb{R}^{n}$, endowed with the maximum norm. This result has been extended later to much more general cases.

It is worth mentioning that in the complex case it is an open problem whether or not the set of norm attaining operators from $C(K)$ to $C(S)$ is dense in $L(C(K), C(S))$.

Theorem 4.1 ([6, Theorem 2.5]). Let $K$ and $S$ be compact Hausdorff topological spaces. Then the pair $(C(K), C(S))$ has the Bishop-Phelps-Bollobás property for operators in the real case. Moreover, the function given by $\eta(\varepsilon)=\frac{\varepsilon^{2}}{12 \cdot 6^{2}}$ satisfies Definition 1.1 in this case for any spaces $K$ and $S$.

There are also results for concrete classes of operators. For a locally compact Hausdorff topological space $L$, we denote by $C_{0}(L)$ either the space of real or complex continuous functions on $L$ with limit zero at infinity.

The next two results are valid in the real case as well as in the complex case. 
THEOREM 4.2 ([6, Theorem 3.3]). Let $Y$ be a uniformly convex Banach space and $L$ a locally compact Hausdorff topological space. Then the space of compact operators from $C_{0}(L)$ to $Y$ has the Bishop-Phelps-Bollobás property. Indeed the function $\eta$ satisfying Definition 3.8 depends only on the modulus of convexity of $Y$.

TheOrem 4.3 ([6, Theorem 4.2]). Let $X$ be a Banach space and $Y$ be a predual of an $L_{1}$-space. Then the space of compact operators from $X$ to $Y$ has the Bishop-PhelpsBollobás property. Indeed the function $\eta$ satisfying Definition 3.8 does not depend on $X$ or $Y$.

A striking result is the one obtained by Kim in 2013 [49. This paper and the arguments used there have inspired some later results.

TheOREM 4.4 ([49, Corollary 2.6]). The pair $\left(c_{0}, Y\right)$ has the Bishop-Phelps-Bollobás property for operators for any uniformly convex Banach space $Y$.

As a consequence of this result, in case that $Y$ is a real strictly convex Banach space, he obtained that the pair $(X, Y)$ has the BPBp if and only if $Y$ is uniformly convex for $X=c_{0}$ or $X=\ell_{\infty}^{n}$ for some natural number $n \geq 2$ [49, Theorem 2.7]. Recall that a normed space is strictly convex if its unit sphere does not contain proper segments.

The next result is due to Kim and Lee and was proved in case that the domain is $L_{\infty}(\mu)$ for some positive measure $\mu$ by Kim, Lee and Lin [52, Theorem 5].

THEOREM 4.5 ([51, Theorem 2.2]). In the real case the pair $(C(K), Y)$ has the BishopPhelps-Bollobás property for operators for any compact Hausdorff topological space $K$, whenever $Y$ is a uniformly convex Banach space.

In order to state an improvement of this result in the complex case, we recall the notion of $\mathbb{C}$-uniformly convex space, introduced by Globevnik [4]. For a complex Banach space $Y$, the $\mathbb{C}$-modulus of convexity $\delta$ is defined for every $\varepsilon>0$ by

$$
\delta(\varepsilon)=\inf \left\{\sup \{\|x+\lambda \varepsilon y\|-1: \lambda \in \mathbb{C},|\lambda|=1\}: x, y \in S_{Y}\right\} .
$$

Recall that the Banach space $Y$ is $\mathbb{C}$-uniformly convex if $\delta(\varepsilon)>0$ for every $\varepsilon>0$ [44]. Every uniformly convex complex space is $\mathbb{C}$-uniformly convex and the converse is not true. Indeed the complex space $L_{1}(\mu)$ is $\mathbb{C}$-uniformly convex [44, Theorem 1] and it is not uniformly convex unless $L_{1}(\mu)$ is one-dimensional. The next result is valid for complex spaces.

TheOREM 4.6 ([3, Theorem 2.4]). The pair $\left(\mathcal{C}_{0}(L), Y\right)$ satisfies the Bishop-Phelps-Bollobás property for operators for any locally compact Hausdorff topological space $L$ and any $\mathbb{C}$-uniformly convex space $Y$. As a consequence, the pair $\left(\mathcal{C}_{0}(L), L_{p}(\mu)\right)$ has the BPBp for any positive measure $\mu$ and $1 \leq p<+\infty$.

The proof of this result is quite technical and uses, amongst other facts, that any operator from $C_{0}(L)$ to a $\mathbb{C}$-uniformly convex space is weakly compact.

From the above result, in the complex case $\left(c_{0}, L_{1}(\mu)\right)$ has the BPBp for any positive measure $\mu$. However, in the real case it is an open problem whether or not the parallel result holds even for the pair $\left(c_{0}, \ell_{1}\right)$. We notice that the set of norm attaining operators 
from $c_{0}$ to $\ell_{1}$ is dense in $L\left(c_{0}, \ell_{1}\right)$ since every operator from $c_{0}$ to $\ell_{1}$ is compact and the usual basis of $c_{0}$ is monotone and shrinking.

For the next results of this section we deal only with real normed spaces unless stated otherwise. It is known that in case that the pair $\left(c_{0}, \ell_{1}\right)$ has the BPBp, then all the pairs $\left(\ell_{\infty}^{n}, \ell_{1}\right)$ have the BPBp "uniformly" (see [20, Theorem 2.1]). This fact motivated the study of the Bishop-Phelps-Bollobás property for operators in case that the domain is $\ell_{\infty}^{n}$. For $n=1$ it is trivially satisfied that the pair $(\mathbb{R}, Y)$ has the Bishop-Phelps-Bollobás property for operators for any Banach space $Y$. In the case $n=2$ the spaces $Y$ such that $\left(\ell_{\infty}^{2}, Y\right)$ has the BPBp are characterized since $\ell_{\infty}^{2}$ is isometric to $\mathbb{R}^{2}$, endowed with the $\ell_{1}$ norm. There is a characterization of those Banach spaces $Y$ such that $\left(\ell_{\infty}^{3}, Y\right)$ has the BPBp (see [9, Theorem 2.9]). That result was extended in [12, Theorem 3.3] for $\ell_{\infty}^{4}$. The general case when the domain is $\ell_{\infty}^{n}$ was considered in [11. In order to recall the intrinsic property appearing in the characterization we need some notation.

For a natural number $n \geq 2$ we define the set $\mathcal{I}_{n}$ by

$$
\mathcal{I}_{n}:=\left\{\left(i_{1}, \ldots, i_{k}\right) \in\{1, \ldots, n\}^{k}: k \text { odd }, k \leq n \text { and } i_{j}<i_{j+1}, \forall j<k\right\} .
$$

If $Y$ is a normed space, we denote by $M_{Y}^{n}$ the set

$$
M_{Y}^{n}:=\left\{\left(y_{i}\right)_{i \leq n} \in Y^{n}: \sum_{j=1}^{k}(-1)^{j+1} y_{i_{j}} \in B_{Y}, \quad \forall\left(i_{1}, \ldots, i_{k}\right) \in \mathcal{I}_{n}\right\} .
$$

The following notion is similar to the definition of approximate hyperplane series property for convex combinations of $n$ elements, but we assume more restrictions in the elements that we approximate.

Definition 4.7 ([11, Definition 2.6]). Let $n \geq 2$ be an integer. A Banach space $Y$ has the approximate hyperplane sum property for $\ell_{\infty}^{n}\left(\mathrm{AHSp}-\ell_{\infty}^{n}\right)$ if for every $0<\varepsilon<1$ there is $0<\gamma_{n}(\varepsilon)<\varepsilon$ satisfying the following condition:

For every $\left(y_{i}\right)_{i \leq n} \in M_{Y}^{n}$, if there exist a nonempty subset $A$ of $\{1, \ldots, n\}$ and $y^{*} \in S_{Y^{*}}$ such that $y^{*}\left(y_{i}\right)>1-\gamma_{n}(\varepsilon)$ for each $i \in A$, then there exists an element $\left(z_{i}\right)_{i \leq n} \in M_{Y}^{n}$ satisfying $\left\|z_{i}-y_{i}\right\|<\varepsilon$ for every $i \leq n$ and $\left\|\sum_{i \in A} z_{i}\right\|=|A|$.

There is a characterization of $\mathrm{AHSp}-\ell_{\infty}^{n}$ that is essential in order to show the next result [11, Proposition 2.9].

TheOREM 4.8. Let $Y$ be a Banach space. The pair $\left(\ell_{\infty}^{n}, Y\right)$ has the Bishop-PhelpsBollobás property for operators if and only if $Y$ has the approximate hyperplane sum property for $\ell_{\infty}^{n}$.

In the proof of the previous characterization [11, Proposition 2.5] the identification of an operator $T \in S_{L\left(\ell_{\infty}^{n}, Y\right)}$ with an element in $M_{Y}^{n}$ is also used. For this identification we associate to each operator its image of a certain basis of $\mathbb{R}^{n}$ whose elements are extreme points in the unit ball of $\ell_{\infty}^{n}$. The proof also gives some estimates between the function $\eta$ appearing in Definition 1.1 and the function $\gamma_{n}$ in Definition 4.7

The class of spaces satisfying the approximate hyperplane sum property for $\ell_{\infty}^{n}$ contains spaces of very different nature (see [11, Section 4]). 
Proposition 4.9. The following classes of Banach spaces satisfy the approximate hyperplane sum property for $\ell_{\infty}^{n}$ for any integer $n \geq 2$ :

1) finite-dimensional spaces,

2) uniformly convex spaces,

3) spaces with property $\beta$ of Lindenstrauss,

4) uniform algebras,

5) $C_{0}(L, Y)$, for any locally compact Hausdorff space $L$, whenever $Y$ is a space with the $A H S p-\ell_{\infty}^{n}$,

6) $L_{1}(\mu)$, for any positive measure $\mu$.

The result for the four first cases is a consequence of the characterization in Theorem 4.8 and some positive result for pairs of Banach spaces with the BPBp. These results can be found in [4, Proposition 2.4], [4, Theorem 5.2], Theorem 2.3 and [26, Theorem 3.6], respectively. The proof of assertion 5) is a consequence in the argument of [9, Proposition 2.4]. In [11, Corollary 4.3] it was proved that the space $\ell_{1}$ has the AHSp- $\ell_{\infty}^{n}$ for any positive integer $n$. This proof is complicated. The result of the general case follows from a simple localization argument. In this case the function $\eta$ such that the pair $\left(\ell_{\infty}^{n}, \ell_{1}\right)$ has the BPBp depends on $n$, so we cannot get that $\left(c_{0}, \ell_{1}\right)$ has the Bishop-Phelps-Bollobás property for operators in the real case.

As we already mentioned in the real case, the pair $\left(c_{0}, Y\right)$ has the Bishop-PhelpsBollobás property for operators if $Y$ has property $\beta$ of Lindenstrauss or if $Y$ is uniformly convex. In order to state a more general result we need the following notion that was used by Lindenstrauss in [57].

Definition 4.10. Let $Y$ be a (real or complex) Banach space, $E \subset S_{Y}$ and $F: E \longrightarrow S_{Y^{*}}$. We recall that the family $E$ is uniformly strongly exposed by $F$ if for every $\varepsilon>0$ there is $\delta>0$ such that

$$
y \in B_{Y}, \quad e \in E, \quad \operatorname{Re} F(e)(y)>1-\delta \Rightarrow\|y-e\|<\varepsilon .
$$

In a uniformly convex space $X$ the set $S_{X}$ is uniformly strongly exposed by $F$, where $F(x) \in\left\{y^{*} \in S_{Y^{*}}: y^{*}(x)=1\right\}$ for any $x \in S_{X}$.

TheOREM 4.11 ([14, Theorem 2.4]). Assume that $Y$ is a (real or complex) Banach space such that there are a set $I,\left\{y_{i}: i \in I\right\} \subset S_{Y},\left\{y_{i}^{*}: i \in I\right\} \subset S_{Y^{*}}$, a subset $E \subset S_{Y}, a$ mapping $F: E \longrightarrow S_{Y^{*}}$ and $0 \leq \rho<1$ satisfying

i) $y_{i}^{*}\left(y_{i}\right)=1, \forall i \in I$.

ii) $\left|y_{i}^{*}\left(y_{j}\right)\right| \leq \rho, \forall i, j \in I, i \neq j$.

iii) $E$ is uniformly strongly exposed by $F$.

iv) $\left|F(e)\left(y_{i}\right)\right| \leq \rho, \forall e \in E, i \in I$.

v) The set $F(E) \cup\left\{y_{i}^{*}: i \in I\right\}$ is a 1-norming set for $Y$; that is, for any $y \in Y$ $\|y\|=\max \left\{\sup \left\{\left|y_{i}^{*}(y)\right|: i \in I\right\}, \sup \{|F(e)(y)|: e \in E\}\right\}$.

Then the pair $\left(c_{0}, Y\right)$ has the BPBp for operators.

As we already mentioned, if $Y$ has property $\beta$ of Lindenstrauss or is uniformly convex, the previous result can be applied. But the class of spaces satisfying the hypothesis of Theorem 4.11 is large as the next result shows. 
TheOrem 4.12 ([14, Corollary 2.8]). Let $Y$ be a uniformly convex Banach space with $\operatorname{dim} X \geq 2$. There is an equivalent norm \|\| on $Y$ arbitrarily close to the original norm of $Y$ and also satisfying the following two conditions:

1) The assumptions of Theorem 4.11.

2) The space $(Y,\|\|)$ neither is uniformly convex nor satisfies property $\beta$ of Lindenstrauss.

Let us mention that the space $Y=\mathbb{R}^{2}$, endowed with the norm whose closed unit ball is the set given by

$$
\operatorname{co}\left\{(x, y) \in \mathbb{R}^{2}: x y \leq 0, x^{2}+y^{2} \leq 1\right\},
$$

is a simple example satisfying the two conditions stated in Theorem 4.12 .

Our purpose now is to state another very interesting result for operators even when the domain can be a space different from $C(K)$. We recall that a uniform algebra is a closed subalgebra $\mathcal{A} \subset C(K)$, endowed with the norm induced by $C(K)$, that separates the points of $K$. The techniques used by the next result are very different from the rest of the results that we mentioned. It uses the $w^{*}$-to-norm fragmentability of the $w^{*}$-compact subsets of the dual of an Asplund space and a Urysohn type result valid for uniform algebras provided by the authors of $[26]$.

Theorem 4.13 ([26, Theorem 3.6 and p. 380]). Let $X$ be a real or complex Banach space, $\mathcal{A} \subset C(K)$ be a uniform algebra and $T \in S_{L(X, \mathcal{A})}$ be an Asplund operator. Assume that $x_{0} \in S_{X}$ satisfies $\left\|T\left(x_{0}\right)\right\|>1-\frac{\varepsilon^{2}}{2}$ for some $0<\varepsilon<\sqrt{2}$. Then there is an Asplund operator $S \in S_{L(X, \mathcal{A})}$ and $z \in S_{X}$ satisfying

$$
\|S(z)\|=1, \quad\|S-T\|<2 \varepsilon \quad \text { and } \quad\left\|z-x_{0}\right\| \leq \varepsilon .
$$

Indeed, the subspaces of finite rank operators, compact operators, weakly compact and Asplund operators in $L(X, \mathcal{A})$ have the BPBp.

The previous result extends the one due to Aron, Cascales and Kozhushkina for $C_{0}(L)$ [18, Corollary 2.5] where the techniques used are similar.

The paper [38] contains several results providing sufficient conditions for the subspace of compact operators between two spaces to have the Bishop-Phelps-Bollobás property for operators. We include here one example of these results.

Theorem 4.14 ([38, Theorem 3.6]). Let $X$ be a Banach space such that $X^{*}$ is isometric to $\ell_{1}$ and $Y$ a Banach space. If the space of compact operators from $c_{0}$ to $Y$ has the Bishop-Phelps-Bollobás property, the space of compact operators from $X$ to $Y$ also satisfies the same property.

By the way, the paper 38] also includes a wide list of pairs of Banach spaces $X$ and $Y$ such that the subspace $K(X, Y)$ (compact operators from $X$ to $Y$ ) has the BishopPhelps-Bollobás property.

5. The Bishop-Phelps-Bollobás property for multilinear mappings. Choi and Song introduced the corresponding version of the BPBp for bilinear forms in [35]. For a family of Banach spaces $X_{1}, \ldots, X_{n}, Y$ we denote by $L^{n}\left(X_{1} \times \ldots \times X_{n}, Y\right)$ the Banach 
space of all continuous $n$-linear mappings from $X_{1} \times \ldots \times X_{n}$ to $Y$. When $Y$ is the scalar field we remove it, i.e. we simply write $L^{n}\left(X_{1} \times \ldots \times X_{n}\right)$. The following notion has been introduced by Choi and Song for bilinear forms [35] and by Dantas, García, Kim, Lee and Maestre for $n$-linear mappings [37, Definition 2.1].

Definition 5.1. Let $X_{1}, X_{2}, \ldots, X_{n}$ and $Y$ be Banach spaces. We say that $\left(X_{1}, \ldots, X_{n} ; Y\right)$ has the Bishop-Phelps-Bollobás property for n-linear mappings (BPBp for $n$-linear mappings, for short) if for any $\varepsilon>0$, there is $\eta(\varepsilon)>0$ such that whenever $A \in S_{L^{n}\left(X_{1} \times \ldots \times X_{N}, Y\right)}$ and $\left(x_{1}, \ldots, x_{n}\right) \in \prod_{i=1}^{n} S_{X_{i}}$ satisfy $\left\|A\left(x_{1}, \ldots, x_{n}\right)\right\|>1-\eta(\varepsilon)$, there are $B \in S_{L^{n}\left(X_{1} \times \ldots \times X_{N}, Y\right)}$ and $\left(z_{1}, \ldots, z_{n}\right) \in \prod_{i=1}^{n} S_{X_{i}}$ such that

$$
\left\|B\left(z_{1}, \ldots, z_{n}\right)\right\|=1, \quad\|B-A\|<\varepsilon \quad \text { and } \quad\left\|z_{i}-x_{i}\right\|<\varepsilon, \forall 1 \leq i \leq n .
$$

If $Y$ is the scalar field we just say that $\left(X_{1}, \ldots, X_{n}\right)$ has the Bishop-Phelps-Bollobás property for n-linear forms.

There are examples of pairs of Banach spaces $(X, Y)$ such that the set of norm attaining bilinear forms on $X \times Y$ is not dense in $L^{2}(X \times Y)$. So these pairs do not satisfy the BPBp for bilinear forms. The following counterexample is due to Choi and Song.

Proposition 5.2 ([35, Theorem 2]). The pair $\left(\ell_{1}, \ell_{1}\right)$ does not satisfy the Bishop-PhelpsBollobás property for bilinear forms.

As a consequence the authors deduce that the same negative result also holds for $n$-linear forms on $\ell_{1}$.

Dai [36] showed that $\left(X_{1}, \ldots, X_{n}\right)$ has the BPBp for $n$-linear forms whenever $\left(X_{1}, \ldots, X_{n+1}\right)$ has the BPBp for $(n+1)$-linear forms and $X_{n+1} \neq\{0\}$.

By using the standard identification of $L^{2}(X \times Y)$ and $L\left(X, Y^{*}\right)$ it is immediate that $\left(X, Y^{*}\right)$ has the BPBp for operators whenever the pair $(X, Y)$ satisfies the BishopPhelps-Bollobás property for bilinear forms. The next result gives a partial converse.

Proposition 5.3 ([10, Proposition 2.4] and [36, Theorem 1.1]). Let $X$ be a Banach space and $Y$ a uniformly convex space. If the pair $\left(X, Y^{*}\right)$ has the Bishop-Phelps-Bollobás property for operators then $(X, Y)$ satisfies the Bishop-Phelps-Bollobás property for bilinear forms.

In general, if $Y$ is a nontrivial space and $\left(X_{1}, \ldots, X_{n} ; Y\right)$ has the BPBp for $n$-linear mappings then $\left(X_{1}, \ldots, X_{n}\right)$ has the BPBp for $n$-linear forms [37, Proposition 2.7]. Dai showed a partial converse of that result in case that $Y$ has property $\beta$ of Lindenstrauss [36]. As a consequence, he obtained that $\left(\ell_{1}, \ell_{p} ; Y\right)$ for $1<p<\infty$ and $\left(\ell_{2}, \ell_{2} ; Y\right)$ has the Bishop-Phelps-Bollobás property for bilinear mappings when $Y$ satisfies property $\beta$.

Now we will state some positive results about the BPBp for multilinear forms. We begin with a result proved by Acosta, Becerra-Guerrero, García and Maestre.

THEOREM 5.4 ([10, Theorem 2.2]). If $X_{i}$ is a uniformly convex Banach space for $1 \leq i \leq n$ then $\left(X_{1}, X_{2}, \ldots, X_{n} ; Y\right)$ satisfies the Bishop-Phelps-Bollobás property for $n$-linear mappings for any Banach space $Y$.

Besides the use of the geometric assumption on the spaces the proof of the previous theorem uses the Stegall perturbed optimization principle. 
The paper [37] contains more results on the topic, including a section devoted to BPBp for polynomials. Another section dealing with this topic for polynomials can be found in [7].

Proposition 5.5 ([37, Proposition 2.3]). Let $X_{i}$ be a finite-dimensional normed space for $1 \leq i \leq n$ and $Y$ a Banach space. Then $\left(X_{1}, \ldots, X_{n} ; Y\right)$ has the Bishop-Phelps-Bollobás property for n-linear mappings.

Theorem 5.6 ([37, Theorem 2.9]). Assume that $X_{i}$ is a Banach space for $1 \leq i \leq n$ and $Y$ is a Banach space whose dual is an $L_{1}$-space. If $\left(X_{1}, \ldots, X_{n}\right)$ has the BishopPhelps-Bollobás property for n-linear forms, then $\left(X_{1}, \ldots, X_{n} ; Y\right)$ has the BishopPhelps-Bollobás property for compact n-linear mappings.

The paper [10] also contains a characterization of those Banach spaces $Y$ such that the pair $\left(\ell_{1}, Y\right)$ has the BPBp for bilinear forms. The property appearing in this characterization implies that $Y^{*}$ has the AHSp.

Definition 5.7 ([10, Definition 3.1]). For a Banach space $Y$ we will say that the pair $\left(Y, Y^{*}\right)$ satisfies the Approximate Hyperplane Series property (shortly AHSp) if for every $\varepsilon>0$ there are $0<\delta, \eta<\varepsilon$ satisfying: for every convex series $\sum_{n} \alpha_{n}$ and for every sequence of functionals $\left\{y_{n}^{*}\right\}$ in $S_{Y^{*}}$ and $y_{0} \in S_{Y}$ such that $\operatorname{Re} \sum_{n} \alpha_{n} y_{n}^{*}\left(y_{0}\right)>1-\eta$, there are a subset $C \subset \mathbb{N},\left\{z_{k}^{*}: k \in C\right\} \subset S_{Y^{*}}$ and $z_{0} \in S_{Y}$ such that

$$
\sum_{k \in C} \alpha_{k}>1-\delta, \quad\left\|z_{k}^{*}-y_{k}^{*}\right\|<\varepsilon, \quad z_{k}^{*}\left(z_{0}\right)=1, \quad \text { for all } \quad k \in C \quad \text { and } \quad\left\|z_{0}-y_{0}\right\|<\varepsilon .
$$

By using similar ideas for the characterization of BPBp for operators whose domain is $\ell_{1}$ the following characterization is proved.

Theorem 5.8 ([10, Theorem 3.6]). Let $Y$ be a Banach space. Then $\left(\ell_{1}, Y\right)$ has the Bishop-Phelps-Bollobás property for bilinear forms if and only if the pair $\left(Y, Y^{*}\right)$ satisfies the Approximate Hyperplane Series property.

As a consequence, the authors of [10] provide several classes of examples in Section 3. Here we include a list of such spaces:

1) uniformly smooth spaces,

2) finite-dimensional spaces,

3) $C_{0}(L)$, for any locally compact Hausdorff topological space $L$,

4) the space of compact operators on any Hilbert space.

However, the pair $\left(L_{1}(\mu), L_{1}(\mu)^{*}\right)$ does not have the AHSp [10, Proposition 4.8] whenever $L_{1}(\mu)$ is infinite-dimensional. We notice that $L_{\infty}(\mu)$ always has the approximate hyperplane series property.

In the case that $X=L_{1}(\mu)$ in general there is no characterization of the spaces $Y$ such that $(X, Y)$ has the Bishop-Phelps-Bollobás property for bilinear forms. But at least it is known under some isomorphic condition on $Y$.

Proposition 5.9 ([7, Theorem 2.4]). Let $Y$ be a Banach space, $\mu$ a positive measure and assume that $L_{1}(\mu)$ is infinite-dimensional. Then $\left(Y, Y^{*}\right)$ has the AHSp whenever $\left(L_{1}(\mu), Y\right)$ has the BPBp for bilinear forms. 
THEOREM 5.10 ([7, Theorem 2.6]). Let $\mu$ be a $\sigma$-finite measure and assume that $L_{1}(\mu)$ is infinite-dimensional and $Y$ an Asplund space. Then the pair $\left(L_{1}(\mu), Y\right)$ has the BishopPhelps-Bollobás property for bilinear forms if and only if $\left(Y, Y^{*}\right)$ has the approximate hyperplane series property.

If $\mu$ is a $\sigma$-finite measure, for the following Banach spaces $X$ the pair $\left(L_{1}(\mu), X\right)$ has the BPBp for bilinear forms (see [7, Corollary 2.7]):

1) finite-dimensional normed spaces,

2) the space $c_{0}$,

3) if $X$ is smooth and $L_{1}(\mu)$ is infinite-dimensional, then $\left(L_{1}(\mu), X\right)$ has the BishopPhelps-Bollobás property for bilinear forms if and only if $X$ is uniformly smooth.

Kim, Lee and Martín proved the following result. The parallel result in the real case is an open problem even for $c_{0}$.

THEOREM 5.11 ([56. Theorem 2]). Let $L_{1}$ and $L_{2}$ be locally compact Hausdorff topological spaces. Then the pair $\left(C_{0}\left(L_{1}\right), C_{0}\left(L_{2}\right)\right)$ has the Bishop-Phelps-Bollobás property for bilinear forms in the complex case.

Some more examples of pairs with the BPBp for bilinear forms can be found in [17. Section 4].

There are also a few results on the same topic for specific classes of bilinear forms or even for sesquilinear forms on a Hilbert space. As an example, we mention the following results, that can be found in [41, Theorems 3.2 and 3.4] and [41, Corollary 2.2].

THEOREM 5.12. Let $H$ be a Hilbert space.

1) The space of symmetric bilinear forms on $H$ satisfies the Bishop-Phelps-Bollobás property both in the real and in the complex case.

2) If $H$ is a complex Hilbert space, the parallel result also holds for the space of symmetric sesquilinear forms.

6. Results for numerical radius. In 2013 Guirao and Kozhushkina studied versions of a Bishop-Phelps-Bollobás theorem for numerical radius of operators [46]. We recall some concepts.

If $X$ is a Banach space and $T \in L(X)$, the numerical radius of $T, \nu(T)$, is defined as

$$
\nu(T)=\sup \left\{\left|x^{*}(T(x))\right|: x \in S_{X}, x^{*} \in S_{X^{*}}, x^{*}(x)=1\right\} .
$$

In general the numerical radius is a semi-norm on $L(X)$ satisfying $\nu(T) \leq\|T\|$ for each $T \in L(X)$. The numerical index of $X, n(X)$ is defined as

$$
n(X)=\inf \left\{\nu(T): T \in S_{L(X)}\right\} .
$$

Hence, $n(X)$ is the greatest constant $t$ such that $t\|T\| \leq \nu(T)$ for each $T \in L(X)$. We always have $0 \leq n(X) \leq 1$ and, in case $n(X)=1$, it is said that $X$ has numerical index equal to 1 . In this case $\nu(T)=\|T\|$ for each $T \in L(X)$. It is well known that the spaces $L_{1}(\mu)$ and $C(K)$ have numerical index equal to 1 for any measure $\mu$ and any compact Hausdorff space $K$ [39, Theorem 2.2]. 
Guirao and Kozhushkina introduced the definition of the BPBp- $\nu$ [46, Definition 1.2]. Afterwards a slightly different concept was introduced by admitting subclasses of the space of bounded linear operators on a Banach space $X$ [13, Definition 2.1]. We recall these notions.

Definition 6.1. Let $X$ be a Banach space and $\mathcal{M}$ a subspace of $L(X)$. We say that $\mathcal{M}$ has the Bishop-Phelps-Bollobás property for numerical radius (BPBp- $\nu$ ) if for every $0<\varepsilon<1$, there is $\eta(\varepsilon)>0$ such that whenever $S \in \mathcal{M}, \nu(S)=1, x_{0} \in S_{X}$ and $x_{0}^{*} \in S_{X^{*}}$ are such that $x_{0}^{*}\left(x_{0}\right)=1$ and $\left|x_{0}^{*}\left(S\left(x_{0}\right)\right)\right|>1-\eta(\varepsilon)$, there are $T \in \mathcal{M}, x_{1} \in S_{X}$ and $x_{1}^{*} \in S_{X^{*}}$ such that

i) $x_{1}^{*}\left(x_{1}\right)=1$

ii) $\left|x_{1}^{*}\left(T\left(x_{1}\right)\right)\right|=\nu(T)=1$,

iii) $\nu(T-S)<\varepsilon,\left\|x_{1}-x_{0}\right\|<\varepsilon$ and $\left\|x_{1}^{*}-x_{0}^{*}\right\|<\varepsilon$.

If the previous property is satisfied for $\mathcal{M}=L(X)$ we say that $X$ has the Bishop-PhelpsBollobás property for numerical radius (BPBp- $\nu$ for short).

The BPBp- $\nu$ is not satisfied for every Banach space. Guirao and Kozhushkina obtained the first result for spaces satisfying the previous property.

Proposition 6.2 ([46, Theorems 5.2 and 5.3]). For any set $\Gamma$ the spaces $\ell_{1}(\Gamma)$ and $c_{0}(\Gamma)$ have the Bishop-Phelps-Bollobás property for numerical radius in the real and in the complex case.

Avilés, Guirao and Rodríguez obtained a result valid for some $C(K)$ spaces in the real case. Although they proved a more general result (see [21, Theorem 3.2]) we state the following special case.

Theorem 6.3 ([21, Corollary 3.3]). The space $C(K)$ has the BPBp- $\nu$ for any metric compact space $K$.

Falcó proved that the space $L_{1}(\mathbb{R})$ also satisfies the Bishop-Phelps-Bollobás property for numerical radius in the real case [40, Theorem 9]. For the proof of this result he used the partition of the real line into dyadic intervals. He also stated in the last section of the same paper that the result is also valid for $L_{1}\left(\mathbb{R}^{n}\right)$ for any positive integer $n$. The next result extends the one just mentioned.

TheOREM 6.4 ([53, Theorem 9]). For any positive measure $\mu$ the real or complex space $L_{1}(\mu)$ has the $B P B p-\nu$.

Kim, Lee and Martín also provided more classes of Banach spaces with the same property and a very general result from the isomorphic point of view.

ThEOREM 6.5 ([53, Propositions 2 and 4]). The following classes of spaces satisfy the Bishop-Phelps-Bollobás property for numerical radius:

1) finite-dimensional normed spaces,

2) Banach spaces that are uniformly convex and uniformly smooth. 
The result stated in 1) uses a compactness argument. To prove the second a convergent sequence of operators is defined by using perturbations of the original operator by rankone operators. By using the uniform convexity of the space and its dual it can be proved that the limit of the sequence of operators attains its numerical radius.

As a consequence of 2 ), the spaces $L_{p}(\mu)$ have the BPBp- $\nu$ for $1<p<\infty$.

THEOREM 6.6 ([53, Theorem 17]). Every infinite-dimensional and separable space is isomorphic to a space with the BPBp- $\nu$.

Kim, Lee and Martín also found some relation between BPBp- $\nu$ and the BPBp for operators under certain restrictions (see for instance [55, Corollary 2.2]).

We finish this section with a result obtained by Acosta, Fakhar and SoleimaniMourchehkhorti. For a result valid for some other classes of subspaces of operators that are not stated here see [13, Theorem 2.1].

THEOREM 6.7 ([13, Corollary 2.1]). Let $\mu$ be a $\sigma$-finite measure. The following subspaces of $L\left(L_{1}(\mu)\right)$ have the Bishop-Phelps-Bollobás property for numerical radius:

1) The space of all finite-rank operators on $L_{1}(\mu)$.

2) The subspace of all compact operators on $L_{1}(\mu)$.

3) The space of all weakly compact operators on $L_{1}(\mu)$.

In the proof of the above result it is used that all operators considered are representable and that the previous subspaces are stable under composition with all continuous operators.

7. Some open problems. Even though there have been published a certain number of papers about the topic in the last years, many problems remain open. Here are some of them.

OPEN QUESTIONS.

1) Does the pair $\left(c_{0}, \ell_{1}\right)$ have the Bishop-Phelps-Bollobás property for operators in the real case?

2) Characterize those Banach spaces $Y$ such that the pair $\left(L_{1}([0,1], Y)\right.$ has the BishopPhelps-Bollobás property for operators.

3) In the complex case, does the pair $(C(K), C(S))$ have the BPBp?

4) Does the space $C(K)$ have the Bishop-Phelps-Bollobás property for numerical radius?

Acknowledgments. The author was supported by Junta de Andalucía grant FQM-185 and also by Spanish MINECO/FEDER grants MTM2015-65020-P and PGC2018-093794B-I00. She also thanks the referees for very careful reading and improving the style of the paper.

\section{References}

[1] M. D. Acosta, Norm attaining operators into $L_{1}(\mu)$, in: Function Spaces (Edwardsville, 1998), Contemp. Math. 232, Amer. Math Soc., Providence, RI, 1999, 1-11. 
[2] M. D. Acosta, Denseness of norm attaining mappings, RACSAM Rev. R. Acad. Cienc. Exactas Fís. Nat. Ser. A Mat. 100 (2006), 9-30.

[3] M. D. Acosta, The Bishop-Phelps-Bollobás property for operators on $\mathcal{C}(K)$, Banach J. Math. Anal. 10 (2016), 307-319.

[4] M. D. Acosta, R. M. Aron, D. García, M. Maestre, The Bishop-Phelps-Bollobás theorem for operators, J. Funct. Anal. 254 (2008), 2780-2799.

[5] M. D. Acosta, R. M. Aron, F. J. García-Pacheco, The approximate hyperplane series property and related properties, Banach J. Math. Anal. 11 (2017), 295-310.

[6] M. D. Acosta, J. Becerra-Guerrero, Y. S. Choi, M. Ciesielski, S. K. Kim, H. J. Lee, M. L. Lourenço, M. Martín, The Bishop-Phelps-Bollobás property for operators between spaces of continuous functions, Nonlinear Anal. 95 (2014), 323-332.

[7] M. D. Acosta, J. Becerra-Guerrero, Y. S. Choi, D. García, S. K. Kim, H. J. Lee, M. Maestre, The Bishop-Phelps-Bollobás property for bilinear forms and polynomials, J. Math. Soc. Japan 66 (2014), 957-979.

[8] M. D. Acosta, J. Becerra-Guerrero, D. García, S. K. Kim, M. Maestre, Bishop-PhelpsBollobás property for certain spaces of operators, J. Math. Anal. Appl. 414 (2014), 532-545.

[9] M. D. Acosta, J. Becerra-Guerrero, D. García, S. K. Kim, M. Maestre, The Bishop-PhelpsBollobás property: a finite-dimensional approach, Publ. Res. Inst. Math. Sci. 51 (2015), 173-190.

[10] M. D. Acosta, J. Becerra-Guerrero, D. García, M. Maestre, The Bishop-Phelps-Bollobás theorem for bilinear forms, Trans. Amer. Math. Soc. 365 (2013), 5911-5932.

[11] M. D. Acosta, J. L. Dávila, A basis of $\mathbb{R}^{n}$ with good isometric properties and some applications to denseness of norm attaining operators, preprint, arXiv:1811.08387v1 [math.FA]

[12] M. D. Acosta, J. L. Dávila, M. Soleimani-Mourchehkhorti, Characterization of the Banach spaces $Y$ satisfying that the pair $\left(\ell_{\infty}^{4}, Y\right)$ has the Bishop-Phelps-Bollobás property for operators, J. Math. Anal. Appl. 470 (2019), 690-715.

[13] M. D. Acosta, M. Fakhar, M. Soleimani-Mourchehkhorti, The Bishop-Phelps-Bollobás property for numerical radius of operators on $L_{1}(\mu)$, J. Math. Anal. Appl. 458 (2018), 925-936.

[14] M. D. Acosta, D. García, S. K. Kim, M. Maestre, The Bishop-Phelps-Bollobás property for operators from $c_{0}$ into some Banach spaces, J. Math. Anal. Appl. 445 (2017), 1188-1199.

[15] M. D. Acosta, M. Mastyło, M. Soleimani-Mourchehkhorti, The Bishop-Phelps-Bollobás and approximate hyperplane series properties, J. Funct. Anal. 274 (2018), 2673-2699.

[16] M. D. Acosta, M. Soleimani-Mourchehkhorti, Stability results of properties related to the Bishop-Phelps-Bollobás property for operators, Sci. China Math., to appear, arXiv: 1902.01677v1[math.FA]

[17] L. Agud, J. M. Calabuig, S. Lajara, E. A. Sánchez-Pérez, Differentiability of $L_{p}$ of a vector measure and applications to the Bishop-Phelps-Bollobás property, Rev. R. Acad. Cienc. Exactas Fís. Nat. Ser. A Mat. RACSAM 111 (2017), 735-751.

[18] R. M. Aron, B. Cascales, O. Kozhushkina, The Bishop-Phelps-Bollobás theorem and Asplund operators, Proc. Amer. Math. Soc. 139 (2011), 3553-3560.

[19] R. M. Aron, Y. S. Choi, D. García, M. Maestre, The Bishop-Phelps-Bollobás theorem for $\mathcal{L}\left(L_{1}(\mu), L_{\infty}[0,1]\right)$, Adv. Math. 228 (2011), 617-628.

[20] R. M. Aron, Y. S. Choi, S. K. Kim, H. J. Lee, M. Martín, The Bishop-Phelps-Bollobás version of Lindenstrauss properties A and B, Trans. Amer. Math. Soc. 367 (2015), 60856101. 
[21] A. Avilés, A. J. Guirao, J. Rodríguez, On the Bishop-Phelps-Bollobás property for numerical radius in $C(K)$ spaces, J. Math. Anal. Appl. 419 (2014), 395-421.

[22] E. Bishop, R. R. Phelps, A proof that every Banach space is subreflexive, Bull. Amer. Math. Soc. 67 (1961), 97-98.

[23] B. Bollobás, An extension to the theorem of Bishop and Phelps, Bull. London Math. Soc. 2 (1970), 181-182.

[24] F. F. Bonsall, J. Duncan, Numerical Ranges II, London Math. Soc. Lecture Note Ser. 10, Cambridge Univ. Press, Cambridge, 1973.

[25] J. Bourgain, On dentability and the Bishop-Phelps property, Israel J. Math. 28 (1977), 265-271.

[26] B. Cascales, A. J. Guirao, V. Kadets, A Bishop-Phelps-Bollobás type theorem for uniform algebras, Adv. Math. 240 (2013), 370-382.

[27] L. Cheng, D. Dai, Y. Dong, A sharp operator version of the Bishop-Phelps Theorem for operators from $\ell_{1}$ to $C L$-spaces, Proc. Amer. Math. Soc. 141 (2013), 867-872.

[28] M. Chica, V. Kadets, M. Martín, J. Merí, Further properties of the Bishop-Phelps-Bollobás moduli, Mediterr. J. Math. 13 (2016), 3173-3183.

[29] M. Chica, V. Kadets, M. Martín, J. Merí, M. Soloviova, Two refinements of the BishopPhelps-Bollobás modulus, Banach J. Math. Anal. 9 (2015), 296-315.

[30] M. Chica, V. Kadets, M. Martín, S. Moreno-Pulido, F. Rambla-Barreno, Bishop-PhelpsBollobás moduli of a Banach space, J. Math. Anal. Appl. 412 (2014), 697-719.

[31] Y. S. Choi, S. K. Kim, The Bishop-Phelps-Bollobás theorem for operators from $L_{1}(\mu)$ to Banach spaces with the Radon-Nikodym property, J. Funct. Anal. 261 (2011), 1446-1456.

[32] Y. S. Choi, S. K. Kim, The Bishop-Phelps-Bollobás property and lush spaces, J. Math. Anal. Appl. 390 (2012), 549-555.

[33] Y. S. Choi, S. K. Kim, H. J. Lee, M. Martín, The Bishop-Phelps-Bollobás theorem for operators on $L_{1}(\mu)$, J. Funct. Anal. 267 (2014), 214-242.

[34] Y. S. Choi, S. K. Kim, H. J. Lee, M. Martín, On Banach spaces with the approximate hyperplane series property, Banach J. Math. Anal. 9 (2015), 243-258.

[35] Y. S. Choi, H. G. Song, The Bishop-Phelps-Bollobás theorem fails for bilinear forms on $\ell_{1} \times \ell_{1}$, J. Math. Anal. Appl. 360 (2009), 752-753.

[36] D. Dai, The Bishop-Phelps-Bollobás theorem for bilinear mappings, Adv. Math. (China) 44 (2015), 105-110.

[37] S. Dantas, D. García, S. K. Kim, H. J. Lee, M. Maestre, On the Bishop-Phelps-Bollobás theorem for multilinear mappings, Linear Algebra Appl. 532 (2017), 406-431.

[38] S. Dantas, D. García, M. Maestre, M. Martín, The Bishop-Phelps-Bollobás property for compact operators, Canad. J. Math. 70 (2018), 53-73.

[39] J. Duncan, C. M. McGregor, J. D. Pryce, A. J. White, The numerical index of a normed space, J. London Math. Soc. (2) 2 (1970), 481-488.

[40] J. Falcó, The Bishop-Phelps-Bollobás property for numerical radius on $L_{1}$, J. Math. Anal. Appl. 414 (2014), 125-133.

[41] D. García, H. J. Lee, M. Maestre, The Bishop-Phelps-Bollobás property for Hermitian forms on Hilbert spaces, Q. J. Math. 65 (2014), 201-209.

[42] F. J. García-Pacheco, The AHSP is inherited by E-summands, Adv. Oper. Theory 2 (2017), $17-20$.

[43] F. J. García-Pacheco, S. Moreno-Pulido, The Bishop-Phelps-Bollobás modulus for functionals on classical Banach spaces, Adv. Oper. Theory 4 (2019), 1-23. 
[44] J. Globevnik, On complex strict and uniform convexity, Proc. Amer. Math. Soc. 47 (1975), $175-178$

[45] W. T. Gowers, Symmetric block bases of sequences with large average growth, Israel J. Math. 69 (1990), 129-151.

[46] A. Guirao, O. Kozhushkina, The Bishop-Phelps-Bollobás property for numerical radius in $\ell_{1}(\mathbb{C})$, Studia Math. 218 (2013), 41-54.

[47] V. Kadets, M. Soloviova, A modified Bishop-Phelps-Bollobás theorem and its sharpness, Mat. Stud. 44 (2015), 84-88.

[48] V. Kadets, M. Soloviova, Quantitative version of the Bishop-Phelps-Bollobás theorem for operators with values in a space with the property $\beta$, Mat. Stud. 47 (2017), 71-90.

[49] S. K. Kim, The Bishop-Phelps-Bollobás theorem for operators from $c_{0}$ to uniformly convex spaces, Israel J. Math. 197 (2013), 425-435.

[50] S. K. Kim, H. J. Lee, Uniform convexity and Bishop-Phelps-Bollobás property, Canad. J. Math. 66 (2014), 373-386.

[51] S. K. Kim, H. J. Lee, The Bishop-Phelps-Bollobás property for operators from $C(K)$ to uniformly convex spaces, J. Math. Anal. Appl. 421 (2015), 51-58.

[52] S. K. Kim, H. J. Lee, P. K. Lin, The Bishop-Phelps-Bollobás property for operators from $L_{\infty}(\mu)$ to uniformly convex Banach spaces, J. Nonlinear Convex Anal. 17 (2016), 243-249.

[53] S. K. Kim, H. J. Lee, M. Martín, On the Bishop-Phelps-Bollobás property for numerical radius, Abstr. Appl. Anal. 2014, Art. ID 479208.

[54] S. K. Kim, H. J. Lee, M. Martín, The Bishop-Phelps-Bollobás theorem for operators from $\ell_{1}$ sums of Banach spaces, J. Math. Anal. Appl. 428 (2015), 920-929.

[55] S. K. Kim, H. J. Lee, M. Martín, On the Bishop-Phelps-Bollobás theorem for operators and numerical radius, Studia Math. 233 (2016), 141-151.

[56] S. K. Kim, H. J. Lee, M. Martín, Bishop-Phelps-Bollobás property for bilinear forms on spaces of continuous functions, Math. Z. 283 (2016), 157-167.

[57] J. Lindenstrauss, On operators which attain their norm, Israel J. Math. 1 (1963), 139-148.

[58] J. R. Partington, Norm attaining operators, Israel J. Math. 43 (1982), 273-276. 